\title{
Comparison of Anti-obesity Effects of Spirit Vinegar and Natural Fermented Vinegar Products on the Differentiation of 3T3-L1 Cells and Obese Rats Fed a High-fat Diet
}

\author{
Hee-Kyoung Son ${ }^{1}$, Yeon-Kyoung Kim ${ }^{1}$, Hye Won Shin ${ }^{2}$, Hee Jeong Lim², Byoung-Seok Moon ${ }^{2}$, Jae-Joon Lee ${ }^{1, *}$ \\ ${ }^{1}$ Department of Food and Nutrition, Chosun University, Gwangju, Korea \\ ${ }^{2}$ Foods Research Institute, CJ Cheil Jedang Corp., Suwon, Korea \\ *Corresponding author: leejj80@chosun.ac.kr
}

\begin{abstract}
This study aimed to compare the effects of spirit vinegar and four types of natural fermented vinegar products (apple vinegar, brown rice vinegar, lemon vinegar, and balsamic vinegar) on the differentiation of 3T3-L1 cells and obesity in rats fed a high-fat diet (HFD). Vinegar inhibited the accumulation of lipid droplets during the differentiation of 3T3-L1 cells. Body weight gains, visceral fat pad weights, serum leptin and triglyceride levels, and hepatic lipogenic enzyme mRNA levels were significantly decreased in the HF-vinegar administered groups compared with those in the HF group. Furthermore, oral administration of vinegars significantly reduced the occurrence of fatty liver deposits and steatosis, and inhibited the HF diet-induced increase in adipocyte size. The anti-obesity and lipid-lowering effects were slightly greater in the HF-natural fermented vinegar groups than in the HF-spirit vinegar group. Apple vinegar had the strongest anti-obesity effects in 3T3-L1 cells and obese rats compared with the other tested vinegars. These findings indicated that vinegar administration may have potential for improving some obesity-related parameters in 3T3-L1 cells and obese rats.
\end{abstract}

Keywords: natural fermented vinegar, spirit vinegar, 3T3-L1 cells, differentiation, high-fat diet, anti-obesity

Cite This Article: Hee-Kyoung Son, Yeon-Kyoung Kim, Hye Won Shin, Hee Jeong Lim, Byoung-Seok Moon, and Jae-Joon Lee, "Comparison of Anti-obesity Effects of Spirit Vinegar and Natural Fermented Vinegar Products on the Differentiation of 3T3-L1 Cells and Obese Rats Fed a High-fat Diet.” Journal of Food and Nutrition Research, vol. 5, no. 8 (2017): 594-605. doi: 10.12691/jfnr-5-8-10.

\section{Introduction}

Obesity is a disease caused by an increase in body fat tissue and the hypertrophy of adipocytes. Its various causes include unbalanced energy intake and consumption, genetic abnormalities, hormone abnormalities, and irregular lifestyle. Obesity has a direct correlation with cardiovascular diseases, cancer, and metabolic diseases such as fatty liver, hyperlipidemia, arteriosclerosis, hypertension, diabetes, and joint disorders induced by an imbalance in the metabolism of lipid and carbohydrates [1]. Prevention of obesity is achieved through dietary control; increased energy expenditure; inhibition of the proliferation, degradation, and accumulation of adipocytes; and degradation of accumulated body fats [2]. Although medications for managing obesity have been developed, these cause side effects such as gastrointestinal distress, abdominal distension, dry mouth, constipation, and hypertension. Thus, attempts have been made to find functional materials from natural substances to manage weight effectively and safely with fewer side effects [3].
Vinegar is a typical fermented food with a unique aroma and sour taste that contains small amounts of volatile and nonvolatile organic acids, sugars, amino acids, and esters $[4,5]$. Since ancient times, vinegar has been widely used in Korea not only for seasoning but also for detoxification and recovery from fatigue and hangovers in the private sector [6]. Vinegar is classified into different types: fermented vinegar, which is prepared from cereal, fruit, and liquor, and synthetic acetic acid vinegar, which is made by diluting glacial acetic acid or acetic acid with drinking water [7]. Spirit vinegar is made using a fermentation process of the dilute of distilled alcohol and contains a higher acidic content (5-20\%) [8], and is used in cooking, pickling, baking, and meat preservation as well as for medicinal, cleaning purposes, and laboratory [9]. Vinegar fermented from fruit or cereal has a lower acidity than spirit vinegar or synthetic vinegar, but it has a pleasing taste and is high in nutritional and various physiologically active substances [5]. Natural fermented vinegars are rich in bioactive compounds such as phenolic compounds, flavonid compounds, carotenoids, phytosterols, amino acids, vitamin E, and vitamin C [5,10-15]. The physiological activities of natural fermented vinegar have 
been reported have effects that include managing constipation [16], diabetes [17,18], obesity [14,19,20,21,22], inflammation [22], hypertension [23], immunity [24], lipid metabolism [19,20], and fatigue [25]. Additionally, many studies have reported that acetic acid, the key component of vinegar and contained, exhibits anti-obesity properties and improves lipid metabolism in animal models [17,19,26,27]. However, no study has been performed to compare the anti-obesity effects of spirit vinegar and natural fermented vinegar in rats.

Therefore, this study was conducted to investigate the inhibitory effects of vinegars on the differentiation of 3T3-L1 cells and their anti-obesity effects in rats fed high-fat diets (HFDs). We compared the anti-obesity effects of spirit vinegar, which mainly comprises acetic acid, with those of natural fermented vinegars (apple, lemon, balsamic, and brown rice vinegars), which are known to contain a large amount of physiologically active substances [10,28,29,30,31].

\section{Materials and Methods}

\subsection{Materials}

The vinegars used in this study included spirit vinegar, balsamic vinegar, and three types of vinegars from CJ CheilJedang Corp. (Seoul, Korea). The natural fermented vinegars manufactured by CJ CheilJedang were fermented through two sequential stages: $1^{\text {st }}$ stage, alcoholic fermentation, and $2^{\text {nd }}$ stage, acetous fermentation. Spirit vinegar was prepared by thinly diluting alcohol with refined water, artificially blowing in air required for acetic acid fermentation, and fermenting it for a few days. The vinegars are produced and commercially available in Korea or abroad.

\subsection{Total Acidity, pH, Sugar, Organic Acids, Total Polyphenolic and Flavonoid Compounds Contents of Vinegar Samples}

Total titratable acidity of vinegar samples was measured by titrating the diluted sampe with $0.1 \mathrm{~N} \mathrm{NaOH}$ until $\mathrm{pH} 8.3$ and expressed as a quantity of acetic acid. $\mathrm{pH}$ was measured using $\mathrm{pH}$ meter (Mettler-Toledo GmbH, Schwerzenbach, Switzerland). Sugar contents were investigated using a automatic digital saccharimeter (Atago pocket PAL-3, Atago Co., Japan). A high-performance liquid chromatography (Shimazu, Kyoto, Japan) was used for the identification and quantification of organic acids in vinegar samples. The total polyphenolic compounds content of vinegar samples were determined according to the methods from Folin-Ciocalteu method [32] with a slightly modification and measured using a UV-visible spectrophotometer (DU-650, Beckman Coulter, Fullerton, CA, USA) at a wavelength of $700 \mathrm{~nm}$. The measurement was calculated using a standard curve of gallic acid $(0-200 \mu \mathrm{g} / \mathrm{mL})$ and expressed as milligrams of gallic acid equivalents (GAE) per liter. The total falvonoid content of vinegar products were determined according to the method of Brimson et al. [32]. The absorbance was measured at $420 \mathrm{~nm}$ using a UV-visible spectrophotometer (DU-650, Beckman Coulter, Fullerton, CA, USA). Flavonoid quantification was based on a standard curve of rutin $(0-200 \mu \mathrm{g} / \mathrm{mL})$. All assays were performed in triplicate.

\subsection{Cell Viability Assay}

3T3-L1 preadipocytes were plated in a 96 well plate at a concentration of $1 \times 10^{4}$ cells/well, cultured in a $5 \% \mathrm{CO}_{2}$ incubator at $37^{\circ} \mathrm{C}$, and diluted with a set concentration of vinegar $(1 / 50,1 / 100,1 / 200,1 / 500,1 / 1000)$, followed by culturing for another $24 \mathrm{~h}$. After completion of culture, $100 \mu \mathrm{g}$ of MTT reagent at a concentration of $5 \mu \mathrm{g} / \mathrm{mL}$ was added, followed by culturing in a $5 \% \mathrm{CO}_{2}$ incubator at $37^{\circ} \mathrm{C}$ for $4 \mathrm{~h}$. After removing the medium, $100 \mu \mathrm{L}$ of DMSO was added to dissolve the generated formazan, and the absorbance was measured at $540 \mathrm{~nm}$ using an ELISA microplate reader (Model 680, Biorad Laboratories Inc., Hercules, CA, USA).

\subsection{Cell Culture and Differentiation}

3T3-L1 preadipocytes (ATCC) were cultured in Dulbecco’s modified Eagle's medium (DMEM, Corning, Manassas, VA, USA) containing $10 \%$ fetal bovine serum (FBS), 100 unit/mL penicillin, and $100 \mathrm{ng} / \mathrm{mL}$ streptomycin in a $5 \% \mathrm{CO}_{2}$ incubator at $37^{\circ} \mathrm{C}$. The cells were dispensed and cultured in a 6 well plate at a density of $6.0 \times 10^{3}$ cell $/ \mathrm{mL}$; when the cells reached confluency, they were cultured in DMEM containing $0.5 \mathrm{mM}$ 3-isobutyl-1-methylxanthine, $5 \mu \mathrm{g} / \mathrm{ml}$ insulin, and $0.25 \mu \mathrm{M}$ dexamethasone for $48 \mathrm{~h}$ to induce differentiation. Subsequently, differentiation was induced by replacing the medium with DMEM containing only $10 \%$ FBS and $5 \mu \mathrm{g} / \mathrm{mL}$ insulin and incubating for $4 \mathrm{~d}$ and then incubating in 10\% FBS/antibiotics/DMEM for 2 d. After $6 \mathrm{~d}$ of inducing differentiation, the cells and culture media were collected and used for the experiments.

\subsection{Oil Red O staining of 3T3-L1 Cells}

The ability of 3T3-L1 preadipocytes to differentiate into adipocytes was confirmed by Oil-Red $\mathrm{O}$ staining. Cultured cells were washed with phosphate buffered saline (PBS), fixed with $10 \%$ formalin for $10 \mathrm{~min}$, washed with $\mathrm{PBS}$, treated with Oil-Red $\mathrm{O}$, and stained at room temperature for $1 \mathrm{~h}$. After staining, the staining solution was removed, and the cells were washed with PBS to observe the degree of formation of fat globules within cells using a microscope. Stained fat globules were eluted with isopropyl alcohol, and absorbance was measured at $520 \mathrm{~nm}$ using a Biotek microplate spectrophotometer (Epoch, Biotek instruments, Winooski, VT, USA).

\subsection{Experimental Animals and Diets}

Fifty-six Sprague Dawley male rats aged 5 weeks were obtained from the Central Lab. Animals Inc. (Seoul, Korea) and adapted to a solid assorted feed for 1 week. Seven groups with 10 rats per each group were assigned randomly, and each of them was placed individually into stainless steel cages and raised for 8 weeks. The following experimental groups were established: the normal diet group $(\mathrm{N})$, high-fat diet group (control group, HF), HF diet plus spirit vinegar administered group (HF-A), HF diet plus apple vinegar administered group (HF-B), HFD 
plus brown rice vinegar administered group (HF-C), HF diet plus lemon vinegar administered group (HF-D), and HF diet plus balsamic vinegar administered group (HF-E). The diets used in this experiment were prepared by modifying the AIN-93 purified diet to the standard [33] (Table 1). Based on the amount of daily intake in humans, the amount of vinegar was administered orally at a dose of $1 \mathrm{~mL} / \mathrm{kg} \mathrm{BW/day}$ at the same time every day. Sterile distilled water was orally administered to the normal and control rats. Water and diet were supplied without restriction; the temperature of the feeding room was maintained $18 \pm 2^{\circ} \mathrm{C}$, and the lighting was adjusted to a 12 -h period (08:00 20:00). Body weight and the amount of dietary intake were measured once a week at a constant time. The animal experimental protocol used in this study was approved by the Institutional Animal Care and Use Committee of Chosun University (CIACUC2015-A0028).

Table 1. Composition of the experimental diet (g/kg diet)

\begin{tabular}{lcc}
\hline Diet composition & $\mathrm{N}$ & $\mathrm{HF}$ \\
\hline Casein & 200.00 & 200.00 \\
L-cystine & 3.00 & 3.00 \\
Corn starch & 397.486 & 267.460 \\
Sucrose & 100.00 & 100.00 \\
Dextrose & 132.00 & 132.00 \\
Cellulose & 50.00 & 50.00 \\
Lard & 0.00 & 200.0 \\
Soybean oil & 70.00 & 70.00 \\
Mineral mix ${ }^{1)}$ & 35.00 & 35.00 \\
Vitamin mix & & 10.00 \\
Choline bitartrate & 10.00 & 2.50 \\
t-Butylhydroquinone & 2.50 & 0.04 \\
\hline
\end{tabular}

${ }^{1,2)}$ AIN-93-MX mineral mixture and AIN-93-VX vitamin mixture [33].

\subsection{Measuring Organ Weights and Serum Biochemical Parameters}

All experimental animals were induced to fast for $12 \mathrm{~h}$ after the end of the experiments and then lightly anesthetized with $\mathrm{CO}_{2}$. After opening the abdomen, blood was collected from the abdominal aorta and centrifuged at $1,900 \times g$ for $20 \mathrm{~min}$ to separate the serum. Subsequently, the liver and adipose tissue (i.e., epididymal, mesenteric, retroperitoneal, perirenal, and total white fat pads) were excised, and rinsed with $0.9 \%$ normal saline to remove the remaining blood, and other adherent substances, and then weighed. Serum alanine aminotransferase (ALT), aspartate aminotransferase (AST), alkaline phosphatase (ALP), and lactate dehydrogenase (LDH) activities as well as triglyceride (TG), total cholesterol (TC), and high-density lipoprotein cholesterol (HDL-C) levels were determined using an automated blood biochemistry analyzer (Fuji Dri-Chem 3500, Fujifilm, Tokyo, Japan). Low-density lipoprotein-cholesterol (LDL-C) was calculated using the Friedewald equation [34]. The serum leptin level was analyzed by immunoassay using the mouse leptin Quantikine ELISA kit (R\&D systems, Inc., Minneapolis, MN, USA).

\subsection{Measuring Liver and Adipose Tissues Lipid Contents}

TG and TC levels in the liver and TG level in epididymal adipose tissues were assessed after extracting total lipids by the Folch method [35]. Briefly, to $0.1 \mathrm{~g}$ of tissue, $6 \mathrm{~mL}$ of $\mathrm{CHCl}_{3}-\mathrm{MeOH}(2: 1$, v/v) was added and left to stand for $3 \mathrm{~d}$ in a refrigerated state. Then, $\mathrm{H}_{2} \mathrm{O}$ was added followed by centrifugation at $1,900 \times g$ for $20 \mathrm{~min}$ to obtain the lower lipid layer containing TG and TC for analysis. The TG level was determined using the method of Biggs et al. [36], and the TC level was analyzed by the method of Zlatkis and Zak [37].

\subsection{Hepatic RNA Extraction and Reverse Transcription-polymerase Chain Reaction (RT-PCR) Analysis}

Hepatic total RNA was isolated using an RNeasy mini kit (QIAGEN, Maryland, USA), and complementary DNA (cDNA) was prepared by reverse transcriptase. Gene expression of acetyl CoA carboxylase (ACC), fatty acid synthetase (FAS), and glucose-6-phosphate dehydrogenase (G6PDH) was measured by RT-PCR with synthesized cDNA and primers (Invitrogem, Carsbad, CA, USA). A RT-PCR analysis was performed using the forward 5'-CAACGCCTTCACACCACCTT-3' and reverse primer (5'-AGCCCATTACTTCATCAAAGATCCT-3') for ACC, F (5'-TGCTCCCAGCTGCAAG-3') and R (5'-GTATCC TCGGGACCGGTTAT-3') for FAS, F (5'-GTTTGGCA GCGGCAACTAA-3') and R (5'-GGCATCACCCTGGT ACAACTC-3') for G6PDH, and F (5'-CTCTCCGCAGC AGGATGAC-3') and R (5'-AAACACGTGGCGGAAT TTG-3') for $\beta$-actin. RT-PCR products were electrophoresed on a $2.0 \%$ agarose gel, and amplified DNA bands were detected using ultraviolet light. The intensities of the DNA bands were analyzed using the Carestream MI SE (Carestream Health Inc., Rochester, NY, USA) program.

\subsection{Histological Analysis of Liver and Epididymal Adipose Tissues}

Liver and epididymal adipose tissues were cut into a uniform size, fixed with a $10 \%$ neutral formalin buffered solution, rinsed, dehydrated with ethanol, and embedded in paraffin. After cutting to a thickness of $4 \mu \mathrm{m}$ using a Cryostat microtome (Leica, Wetzlar, Germany), the liver tissue was stained with Oil-Red $\mathrm{O}$, the fat tissue was stained with hematoxylin \& eosin ( $\mathrm{H} \& \mathrm{E})$, and the sections were examined using light microscopy (Olympus D970, Olympus Optical Co., Tokyo, Japan). The size of the adipose tissue was photographed and measured at 400 $\mathrm{X}$ magnification, and Axio-Vision Rel. 4.6 software (Carl Zeiss) was used to compare the cell sizes of the tissues.

\subsection{Statistical Analysis}

All statistical analyses were conducted using SPSS (Statistical Package for Social Science). The results are presented as the mean \pm standard error in each group, and one-way analysis of variance was used to test the 
statistical significance of the mean difference between groups, followed by post hoc testing using Turkey's test at $p<0.05$.

\section{Results}

\subsection{Physicochemical Composition, Total Polyphenolics and Flavonoids Contents}

Total acidity, $\mathrm{pH}$, sugar, organic acids, total polyphenolic and flavonoid compounds contents in vinegar samples are given in Table 2. The organic acid contents were composed of acetic acid, citric acid, succinic acid, lactic acid and acetic acid, with the greatest content of acetic acid in all vinegars. The citric acid content was highest in apple vinegar. The $\mathrm{pH}$ values and total acidity of the vinegar samples were significantly different showing the range $2.32-2.98$ and $5.47-7.08 \%$, respectively. The sugar content of balsamic vinegar was significantly higher than the other vinegars. The total polyphenolic and falvonoid compound contents were detected in the following order; balsamic vinegar $>$ apple vinegar $>$ lemin vinegar $>$ brown rice vinegar $>$ sprit vinegar.

\subsection{Cytotoxicity}

The effect of vinegar on the cell viability of 3T3-L1 preadipocytes was evaluated using the MTT assay. The optimum $\mathrm{pH}$ for cell growth is 6.9-7.8; however, since the organic acid in vinegar affects growth by changing the $\mathrm{pH}$ of the medium, pretreatment of 3T3-L1 preadipocytes with vinegars without $\mathrm{pH}$ adjustment showed cytotoxicity up to $1 / 50$ dilution rate (data not shown). Thus, after adjustment of the $\mathrm{pH}$ of vinegar with $10 \mathrm{~N} \mathrm{NaOH}$ to $\mathrm{pH}$ 7.0 , the vinegar was diluted $(1 / 200,1 / 100,1 / 50,1 / 10)$, as mentioned above. The all vinegar groups showed no toxicity at $24 \mathrm{~h}$ after the pretreatment compared with the control group; in addition, cell viability did not decrease (Figure 1).

Table 2. Physicochemical compositions of spirit and natural fermented vinegar products

\begin{tabular}{|c|c|c|c|c|c|}
\hline & Spirit vinegar & Apple vinegar & Brown rice vinegar & Lemon vinegar & Balsamic vinegar \\
\hline \multicolumn{6}{|l|}{ Organic acids(g/L) } \\
\hline Citric acid & $\mathrm{ND}^{\mathrm{c}}$ & $2.25 \pm 0.01^{\mathrm{a}}$ & $0.12 \pm 0.00^{\mathrm{b}}$ & $\mathrm{ND}^{\mathrm{c}}$ & $\mathrm{ND}^{\mathrm{c}}$ \\
\hline Malic acid & ND & ND & ND & ND & ND \\
\hline Succinic acid & $0.28 \pm 0.00^{1 \mathrm{a} 2)}$ & $0.27 \pm 0.00^{\mathrm{a}}$ & $0.20 \pm 0.00^{\mathrm{b}}$ & $0.02 \pm 0.00^{\mathrm{b}}$ & $0.17 \pm 0.00^{\mathrm{c}}$ \\
\hline Lactic acid & $0.29 \pm 0.01^{\mathrm{b}}$ & $0.15 \pm 0.00^{c}$ & $0.27 \pm 0.00^{\mathrm{b}}$ & $\mathrm{ND}^{\mathrm{d}}$ & $0.43 \pm 0.01^{\mathrm{a}}$ \\
\hline Formic acid & ND & ND & ND & ND & ND \\
\hline Acetic acid & $51.89 \pm 1.13^{\mathrm{a}}$ & $43.24 \pm 0.94^{b}$ & $39.78 \pm 1.20^{c}$ & $46.69 \pm 1.07^{\mathrm{b}}$ & $53.55 \pm 0.93^{\mathrm{a}}$ \\
\hline Total acidity (\%, as acetic acid) & $6.45 \pm 0.11^{\mathrm{c}}$ & $7.08 \pm 0.08^{\mathrm{a}}$ & $6.71 \pm 0.06^{\mathrm{b}}$ & $6.45 \pm 0.03^{\mathrm{c}}$ & $5.47 \pm 0.03^{\mathrm{d}}$ \\
\hline Total sugar $\left({ }^{\circ}\right.$ Brix $)$ & $3.20 \pm 0.00^{c}$ & $6.60 \pm 0.00^{\mathrm{b}}$ & $7.60 \pm 0.00^{\mathrm{b}}$ & $6.10 \pm 0.00^{\mathrm{b}}$ & $25.10 \pm 0.00^{\mathrm{a}}$ \\
\hline $\mathrm{pH}$ & $2.32 \pm 0.00^{c}$ & $2.98 \pm 0.25^{\mathrm{a}}$ & $2.69 \pm 0.03^{\mathrm{b}}$ & $2.97 \pm 0.01^{\mathrm{s}}$ & $2.92 \pm 0.04^{\mathrm{a}}$ \\
\hline $\begin{array}{l}\text { Total polyphenolic compounds } \\
\text { (mg GAE /100 mL) }\end{array}$ & $\mathrm{ND}^{\mathrm{d}}$ & $44.05 \pm 0.53^{\mathrm{b}}$ & $25.98 \pm 1.12^{\mathrm{c}}$ & $30.15 \pm 0.59^{\mathrm{c}}$ & $143.04 \pm 6.93^{\mathrm{a}}$ \\
\hline $\begin{array}{l}\text { Total flavonoid compounds } \\
\text { (mg RE /100 mL) }\end{array}$ & $\mathrm{ND}^{\mathrm{d}}$ & $7.73 \pm 1.08^{b}$ & $3.14 \pm 0.12^{\mathrm{c}}$ & $3.68 \pm 0.17^{c}$ & $109.34 \pm 8.26^{\mathrm{a}}$ \\
\hline
\end{tabular}

${ }^{1)}$ All values are expressed as mean $\pm \mathrm{SE}(\mathrm{n}=3)$..

${ }^{2)}$ Values with different superscript in the same row significantly different $(\mathrm{p}<0.05)$ among groups by Tukey's test.

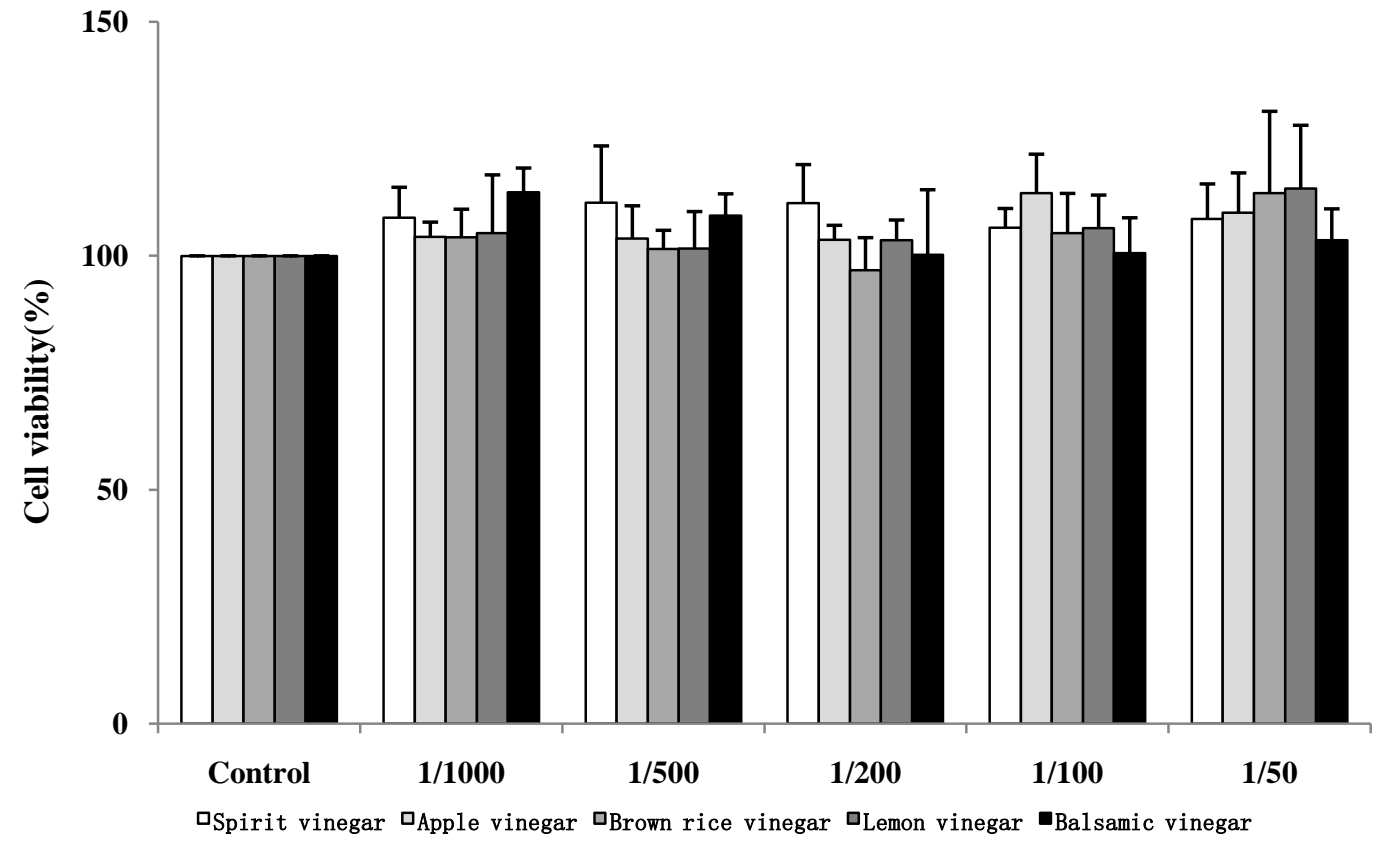

Figure 1. Cytotoxic effects of spirit vinegar and natural fermented vinegar products on 3T3-L1 preadipocytes. 3T3-L1 preadipocytes were treated with vinegars at various dilutions (1/50-1/1000) for $24 \mathrm{~h}$. Cell viability was evaluated with the MTT Assay System. Values are presented as the mean \pm SD $(\mathrm{n}=3)$ 


\subsection{Inhibitory Effects of Spirit Vinegar and Natural Fermented Vinegars on Adipogenesis in 3T3-L1 Cells}

Lipid formation and accumulation in response to non-toxic dilutions of vinegars with $\mathrm{pH}$ adjustment were analyzed by Oil Red O staining to evaluate differentiation into adipocytes (Figure 2). Microscopic observations revealed that the control group was actively induced to form lipid globules within the cytoplasm. The group treated with caffeine $(0.2 \mathrm{mg} / \mathrm{mL})$, which is known to inhibit lipid differentiation [38], showed fewer lipid globules compared to the control group (Figure 2A). The formation of lipid globules in the groups administered vinegar was reduced in a dose-dependent manner. Fat accumulation in 3T3-L1 cells was significantly inhibited only at the dilution of $1 / 50$ in the balsamic vinegar treated group; however, administration of the other vinegars resulted in a significant dose-dependent inhibition of fat accumulation in 3T3-L1 cells (Figure 2B). Among the vinegar treatments, apple vinegar showed the strongest inhibition effect on the differentiation of 3T3-L1 cells.

(A)

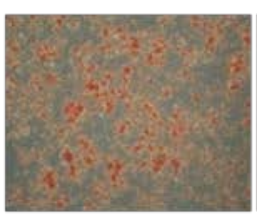

Control

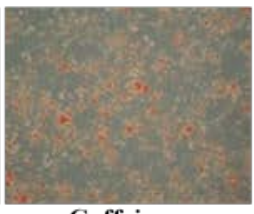

Caffeine

$1 / 200$
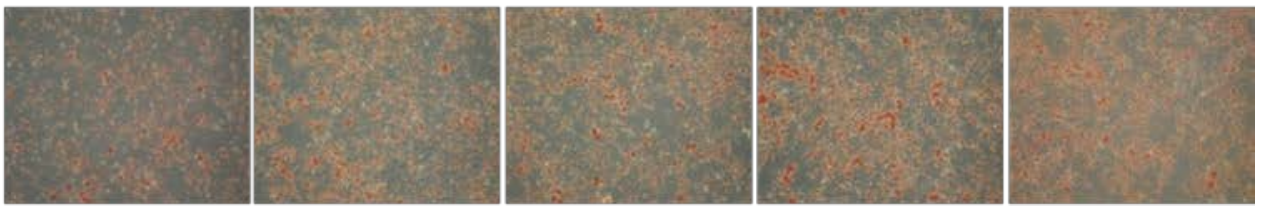

$1 / 100$
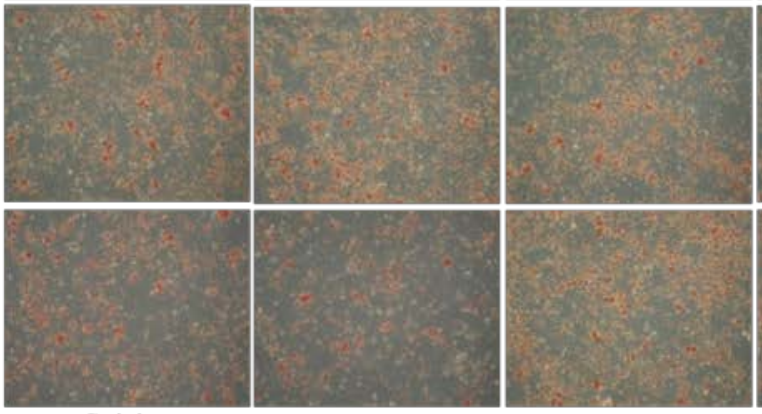

Brown rice vinegar

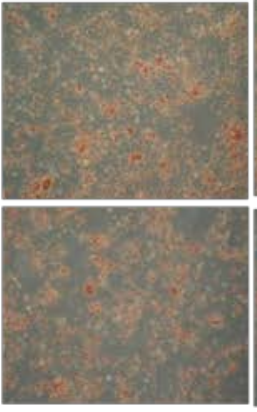

Lemon

vinegar

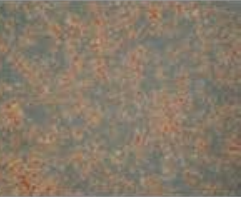

$1 / 50$

(B)

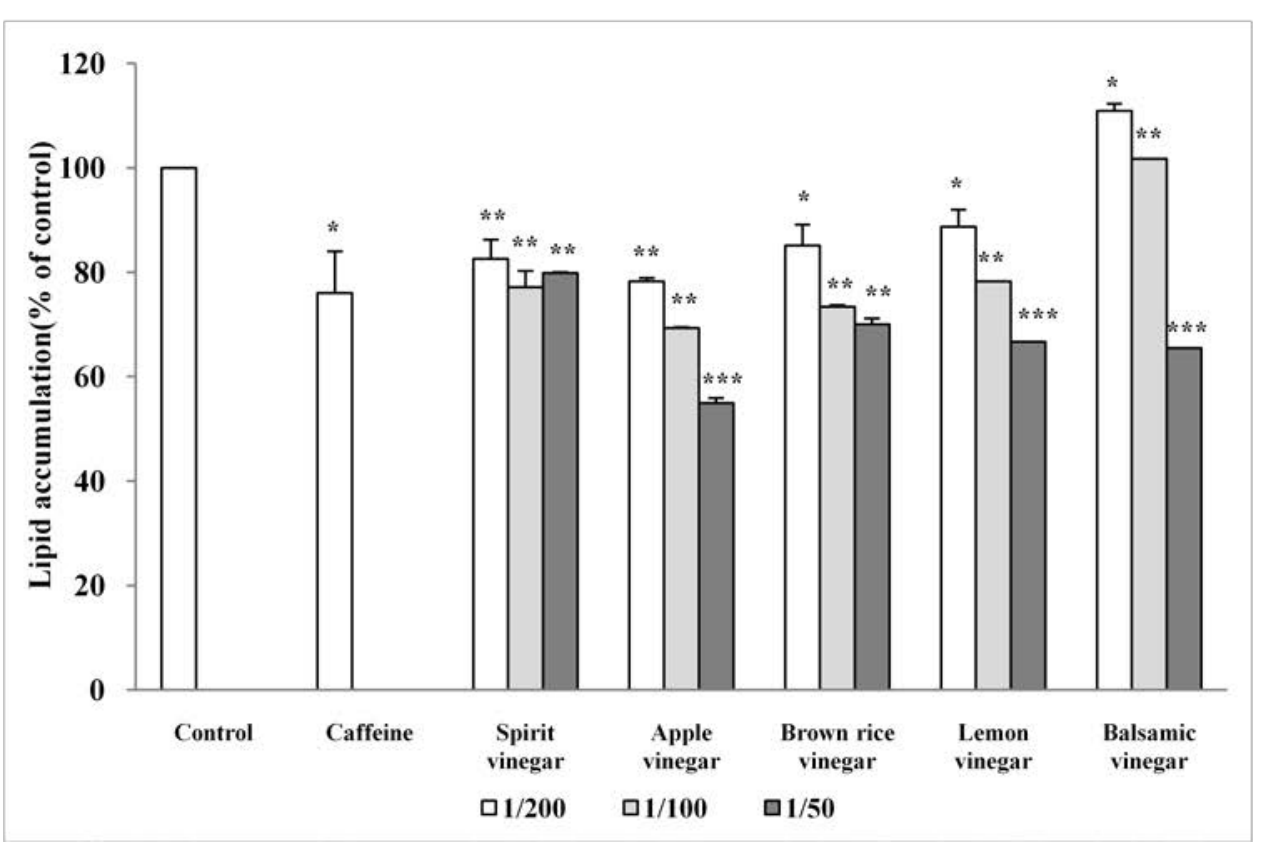

Figure 2. Inhibitory effects of spirit vinegar and natural fermented vinegar products on lipid accumulation in fully differentiated 3T3-L1 adipocytes. 3T3-L1 preadipocytes were differentiated by adding adipogenic inducers (insulin, dexamethasone, and 3-isobutyl-1-methylxanthine) when the cells reached confluence, followed by $2 \mathrm{~d}$ of incubation. Complete differentiation to adipocytes was achieved by further culturing the cells in $10 \%$ FBS/DMEM with $5 \mu \mathrm{g} / \mathrm{mL}$ insulin for $4 \mathrm{~d}$ and then in 10\% FBS/antibiotics/DMEM for $2 \mathrm{~d}$. Vinegars were added during all stages of differentiation. (A) Intracellular triglycerides were stained with Oil Red O. (B) Oil Red O was dissolved with isopropanol, and optical density was measured at $520 \mathrm{~nm}$. Values are presented as the mean $\pm \mathrm{SD}(\mathrm{n}=3) .{ }^{*} \mathrm{p}<0.05,{ }^{* *} \mathrm{p}<0.01,{ }^{* * *} \mathrm{p}<0.001$ compared to the control 
Table 3. Effects of orally administered spirit and natural fermented vinegar products on body weight gain, food intake, and relative tissue weight in rats fed a high-fat diet

\begin{tabular}{lccccccc}
\hline & \multicolumn{7}{c}{ Groups $^{1)}$} \\
\cline { 2 - 7 } & $\mathrm{N}$ & HF & HF-A & HF-B & HF-C & HF-D & HF-E \\
\hline Body weight gain (g/day) & $5.65 \pm 0.22^{3)(4)}$ & $7.12 \pm 0.26^{\mathrm{a}}$ & $6.91 \pm 0.33^{\mathrm{ab}}$ & $6.13 \pm 0.24^{\mathrm{b}}$ & $6.85 \pm 0.30^{\mathrm{ab}}$ & $6.68 \pm 0.37^{\mathrm{ab}}$ & $6.44 \pm 0.36^{\mathrm{b}}$ \\
Food intake (g/day) & $24.72 \pm 0.58^{\mathrm{a}}$ & $20.02 \pm 0.53^{\mathrm{b}}$ & $20.62 \pm 0.63^{\mathrm{b}}$ & $20.10 \pm 0.58^{\mathrm{b}}$ & $20.89 \pm 0.55^{\mathrm{b}}$ & $20.75 \pm 0.56^{\mathrm{b}}$ & $20.95 \pm 0.55^{\mathrm{b}}$ \\
FER $^{2)}$ & $0.23 \pm 0.01^{\mathrm{b}}$ & $0.36 \pm 0.01^{\mathrm{a}}$ & $0.34 \pm 0.01^{\mathrm{a}}$ & $0.31 \pm 0.02^{\mathrm{a}}$ & $0.33 \pm 0.01^{\mathrm{a}}$ & $0.32 \pm 0.01^{\mathrm{a}}$ & $0.31 \pm 0.01^{\mathrm{a}}$ \\
Liver weight (g/100 g body wt) & $2.62 \pm 0.13^{\mathrm{NS})}$ & $2.91 \pm 0.05$ & $2.74 \pm 0.09$ & $2.70 \pm 0.06$ & $2.76 \pm 0.05$ & $2.75 \pm 0.08$ & $2.71 \pm 0.07$ \\
\hline White fat pads (g/100 g body wt) & & & & & & & \\
Epididymal & $2.16 \pm 0.15^{\mathrm{b}}$ & $3.01 \pm 0.19^{\mathrm{a}}$ & $2.69 \pm 0.26^{\mathrm{ab}}$ & $2.30 \pm 0.09^{\mathrm{b}}$ & $2.65 \pm 0.16^{\mathrm{ab}}$ & $2.54 \pm 0.13^{\mathrm{ab}}$ & $2.66 \pm 0.13^{\mathrm{ab}}$ \\
Mesenteric & $1.01 \pm 0.07^{\mathrm{b}}$ & $1.44 \pm 0.10^{\mathrm{a}}$ & $1.35 \pm 0.08^{\mathrm{ab}}$ & $1.23 \pm 0.09^{\mathrm{ab}}$ & $1.28 \pm 0.12^{\mathrm{ab}}$ & $1.27 \pm 0.07^{\mathrm{ab}}$ & $1.28 \pm 0.09^{\mathrm{ab}}$ \\
Retroperitoneal & $2.77 \pm 0.13^{\mathrm{c}}$ & $3.82 \pm 0.12^{\mathrm{a}}$ & $3.62 \pm 0.14^{\mathrm{a}}$ & $3.04 \pm 0.08^{\mathrm{bc}}$ & $3.50 \pm 0.15^{\mathrm{ab}}$ & $3.42 \pm 0.09^{\mathrm{ab}}$ & $3.43 \pm 0.21^{\mathrm{ab}}$ \\
Perirenal & $0.85 \pm 0.06^{\mathrm{b}}$ & $1.15 \pm 0.09^{\mathrm{a}}$ & $0.94 \pm 0.04^{\mathrm{b}}$ & $0.91 \pm 0.05^{\mathrm{b}}$ & $0.92 \pm 0.04^{\mathrm{b}}$ & $0.92 \pm 0.02^{\mathrm{b}}$ & $0.92 \pm 0.04^{\mathrm{b}}$ \\
Total fat pads & $6.79 \pm 0.28^{\mathrm{c}}$ & $9.41 \pm 0.36^{\mathrm{a}}$ & $8.60 \pm 0.30^{\mathrm{ab}}$ & $7.51 \pm 0.18^{\mathrm{bc}}$ & $8.36 \pm 0.25^{\mathrm{ab}}$ & $8.15 \pm 0.22^{\mathrm{b}}$ & $8.29 \pm 0.30^{\mathrm{b}}$ \\
\hline
\end{tabular}

${ }^{11} \mathrm{~N}$ : normal diet group, HF: high-fat diet group, HF-A: high-fat diet + spirit vinegar administered group, HF-B: high-fat diet + apple vinegar administered group, HF-C: high-fat diet + brown rice vinegar administered group, HF-D: high-fat diet + lemon vinegar administered group, HF-E: highfat diet + balsamic vinegar administered group.

${ }^{2)}$ FER (food efficiency ratio): weight gain (g/day)/food intake (g/day).

${ }^{3)}$ The results are the mean \pm SE of 10 rats per each group.

${ }^{4)}$ NS: No significance.

${ }^{5)}$ Values with different superscripts in the same row are significantly different $(\mathrm{p}<0.05)$ among the groups by Tukey's test.

\subsection{Body Weight Gain, Food Intake, and Food Efficiency Ratio (FER) in Rats}

No mortality was observed in any of the experimental groups during the entire experimental period, and the test substances caused no clinical symptoms. The body weight gains of the HF group increased markedly compared with those of the $\mathrm{N}$ group (Table 3). The HF-vinegar administered groups showed lower body weight gains than the HF group; moreover, the HF-B and HF-E groups showed the lowest body weight gain, reaching a similar body weight as the $\mathrm{N}$ group. The food intakes of the HF groups were significantly lower compared with those of the $\mathrm{N}$ group, and there was no significant difference between the HF groups. FER significantly increased in the HF groups compared to the $\mathrm{N}$ group.

\subsection{Liver and White Fat Pad Weights in Rats}

The HF-vinegar administered groups had lower liver weights than the HF group with no statistical significance, and no difference was found with the $\mathrm{N}$ group (Table 3 ). The weights of epididymal, mesenteric, perirenal, and total white fat pads significantly increased in the HF group compared to those in the $\mathrm{N}$ group. Total fat pads weights were lower in the HF-B, HF-D, and HF-E groups compared to the HF group. Moreover, the weights of epididymal, mesenteric, and retroperitoneal fat pads were lower in the HF-vinegar administered groups compared to the HF group. The weight of perirenal fat pads was significantly lower in the HF-vinegar administered groups compared to the HF group. Among the HF-vinegar administered groups, the HF-B group had the lowest epididymal, retroperitoneal, and total fat pad weights.

\subsection{Serum Lipid Profiles and Leptin Contents}

Serum TG, TC, and LDL-C contents were significantly increased in the HF group compared to the $\mathrm{N}$ group (Table 4). Serum TC and LDL-C contents were significantly reduced in the HF-B, HF-C, HF-D, and HF-E groups compared to the HF group. Serum TG levels were slightly lower in the HF-B, HF-C, and HF-E groups than in the HF group. However, significant differences in serum HDL-C contents were not observed among the groups. Among the HF-vinegar administered groups, the HF-B group had the lowest serum levels of TC and LDL-C, and the levels were similar to those in the $\mathrm{N}$ group. Serum leptin levels were significantly higher in the HF group than in the $\mathrm{N}$ group (Table 4). The toxicity of spirit vinegar and natural fermented vinegars was determined by measuring serum ALT, AST, ALP, and LDH activities. Serum ALT, AST, ALP, and LDH activities significantly increased in the HF group compared to those in the $\mathrm{N}$ group. The increase in ALT activity caused by the HF diet was significantly decreased by apple vinegar, lemon vinegar, or balsamic vinegar administration. The HF-vinegar administered groups had lower LDH activities compared to those in the HF group. However, there were no significant differences in serum AST activity between the HF groups. The serum levels of leptin were significantly reduced in the HF-vinegar administered groups compared to those in HF group, and the HF-B, HF-C, HF-D, and HF-E groups showed no significant difference with the $\mathrm{N}$ group.

\subsection{Hepatic TG and TC Contents and Histological Changes}

Hepatic TG and TC contents were significantly increased in the HF group compared to those in the $\mathrm{N}$ group (Figure $3 \mathrm{~A}$ ). The TG contents of the HF-A, HF-B, HF-C, and HF-D groups were significantly decreased compared to those in the HF group. Hepatic TC levels in the HF-vinegar administered groups (HF-B, HF-C, HF-D, and HF-E) except for HF-A were significantly lower than that in the HF group. The macroscopic appearance of the liver photographed immediately after sacrificing the rats (Figure 3B) was blood-red in the $\mathrm{N}$ group and pale pink in the $\mathrm{HF}$ group due to deposited yellow fat. In the HF-vinegar administered groups, the liver was less reddish than in the $\mathrm{N}$ group but had less lipid deposition than that 
in the HF group. In addition, the results of Oil Red O staining (Figure 3C) to confirm hepatic fat accumulation showed that the HF group had marked red staining in hepatocytes compared with the $\mathrm{N}$ group, indicating that fat accumulation was increased. Lipid accumulation in hepatocytes of the HF-vinegar administered groups was suppressed compared to the levels in the HF group. Among the HF-vinegar administered groups, fat accumulation in hepatic tissue was most inhibited in the HF-B group.

Table 4. Effects of orally administered spirit and natural fermented vinegar products on serum lipid profiles, cardiac biomarkers, and leptin levels in rats fed a high-fat diet

\begin{tabular}{|c|c|c|c|c|c|c|c|}
\hline & \multicolumn{7}{|c|}{ Groups $^{1)}$} \\
\hline & $\mathrm{N}$ & $\mathrm{HF}$ & HF-A & HF-B & HF-C & HF-D & HF-E \\
\hline \multicolumn{8}{|c|}{ Serum lipid profiles (mg/dL) } \\
\hline Triglyceride & $61.34 \pm 3.61^{2)(3)}$ & $96.32 \pm 3.32^{\mathrm{a}}$ & $83.10 \pm 3.55^{\mathrm{ab}}$ & $72.54 \pm 2.14^{\mathrm{b}}$ & $72.61 \pm 4.50^{\mathrm{b}}$ & $79.92 \pm 3.70^{\mathrm{b}}$ & $75.25 \pm 2.85^{\mathrm{b}}$ \\
\hline Total cholesterol & $88.31 \pm 4.51^{\mathrm{d}}$ & $130.19 \pm 2.98^{\mathrm{a}}$ & $117.92 \pm 5.65^{\mathrm{ab}}$ & $102.36 \pm 3.59^{\text {cd }}$ & $108.32 \pm 4.63^{\text {bc }}$ & $121.24 \pm 5.09^{\mathrm{ab}}$ & $115.18 \pm 4.84^{\mathrm{ab}}$ \\
\hline HDL-cholesterol & $41.89 \pm 4.43^{\mathrm{NS}}$ & $28.44 \pm 2.90$ & $37.61 \pm 5.76$ & $39.15 \pm 4.55$ & $32.10 \pm 4.55$ & $38.47 \pm 4.89$ & $36.13 \pm 4.51$ \\
\hline LDL-cholesterol & $34.25 \pm 1.86^{c}$ & $84.44 \pm 1.52^{\mathrm{a}}$ & $65.68 \pm 7.88^{\mathrm{ab}}$ & $48.64 \pm 4.71^{\text {bc }}$ & $61.68 \pm 6.35^{\mathrm{b}}$ & $66.82 \pm 5.35^{\mathrm{b}}$ & $63.96 \pm 5.84^{\mathrm{b}}$ \\
\hline \multicolumn{8}{|c|}{ Serum cardiac biomarkers (U/L) } \\
\hline ALT & $153.19 \pm 7.90^{\mathrm{b}}$ & $209.62 \pm 11.48^{\mathrm{a}}$ & $187.11 \pm 6.92^{\mathrm{ab}}$ & $158.73 \pm 8.51^{\mathrm{b}}$ & $178.47 \pm 6.75^{\mathrm{ab}}$ & $154.72 \pm 12.44^{\mathrm{b}}$ & $152.25 \pm 12.88^{\mathrm{b}}$ \\
\hline AST & $26.53 \pm 1.49^{b}$ & $53.67 \pm 4.18^{\mathrm{a}}$ & $41.10 \pm 5.47^{\mathrm{ab}}$ & $39.51 \pm 47.72^{\mathrm{ab}}$ & $42.84 \pm 5.00^{\mathrm{ab}}$ & $40.32 \pm 3.89^{\mathrm{ab}}$ & $37.01 \pm 3.23^{\mathrm{ab}}$ \\
\hline ALP & $427.12 \pm 19.94^{c}$ & $710.60 \pm 27.4^{\mathrm{a}}$ & $582.84 \pm 20.59^{\mathrm{b}}$ & $571.11 \pm 25.04^{\mathrm{b}}$ & $592.94 \pm 23.11^{\mathrm{b}}$ & $608.13 \pm 51.82^{\mathrm{ab}}$ & $588.44 \pm 30.80^{\mathrm{b}}$ \\
\hline LDH & $48.51 \pm 3.96^{c}$ & $102.57 \pm 8.27^{\mathrm{a}}$ & $72.72 \pm 6.47^{\mathrm{b}}$ & $62.50 \pm 4.31^{\mathrm{bc}}$ & $72.64 \pm 3.86^{\mathrm{b}}$ & $62.86 \pm 4.80^{\mathrm{bc}}$ & $71.32 \pm 5.93^{\mathrm{b}}$ \\
\hline Leptin (pg/mL) & $430.42 \pm 30.66^{c}$ & $757.29 \pm 38.35^{\mathrm{a}}$ & $591.29 \pm 36.56^{\mathrm{b}}$ & $487.04 \pm 31.20^{\mathrm{bc}}$ & $538.42 \pm 17.70^{\mathrm{bc}}$ & $507.21 \pm 26.63^{\mathrm{bc}}$ & $250.36 \pm 29.67^{\mathrm{b}}$ \\
\hline
\end{tabular}

${ }^{1)} \mathrm{N}$ : normal diet group, HF: high-fat diet group, HF-A: high-fat diet + spirit vinegar administered group, HF-B: high-fat diet + apple vinegar administered group, HF-C: high-fat diet + brown rice vinegar administered group, HF-D: high-fat diet + lemon vinegar administered group, HF-E: highfat diet + balsamic vinegar administered group.

${ }^{2)}$ The results are the mean \pm SE of 10 rats per each group.

${ }^{3)}$ Values with different superscripts in the same row are significantly different $(\mathrm{p}<0.05)$ among the groups by Tukey's test.

(A)
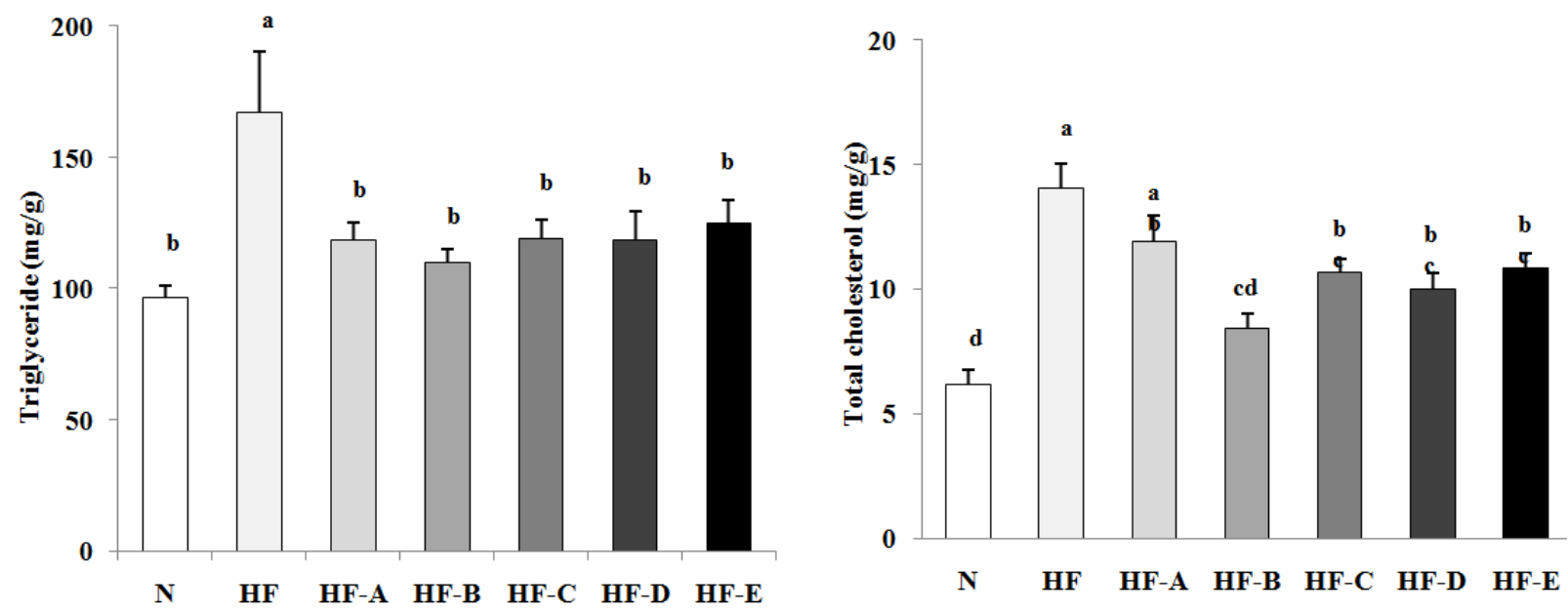

(B)
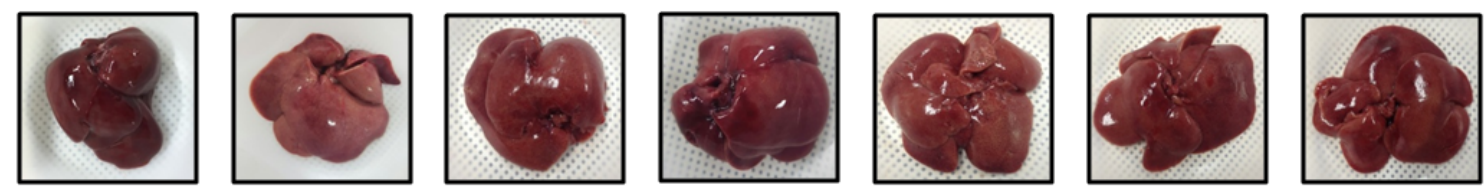

(C)

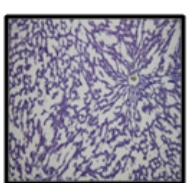

$\mathbf{N}$

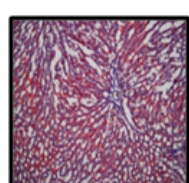

HF

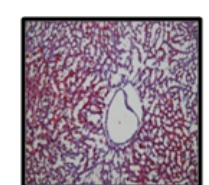

HF-A

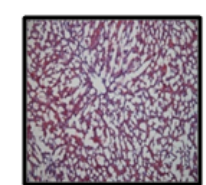

HF-B

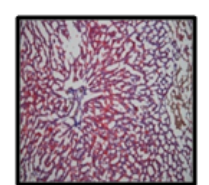

HF-C

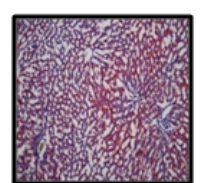

HF-D

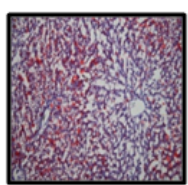

HF-E

Figure 3. Effects of spirit vinegar and natural fermented vinegar products on hepatic triglyceride and total cholesterol contents (A), representative anatomical views (B), and histological analysis (C) in rats fed a high-fat diet. All sections were stained with Oil Red O, $\times 100$. Values are the mean \pm SE $(\mathrm{n}=10)$. Bars with different letters are significantly different at $\mathrm{p}<0.05$ by Tukey's test. N: normal diet group, HF: high-fat diet group, HF-A: highfat diet + spirit vinegar administered group, HF-B: high-fat diet + apple vinegar administered group, HF-C: high-fat diet + brown rice vinegar administered group, HF-D: high-fat diet + lemon vinegar administered group, and HF-E: high-fat diet + balsamic vinegar administered group 


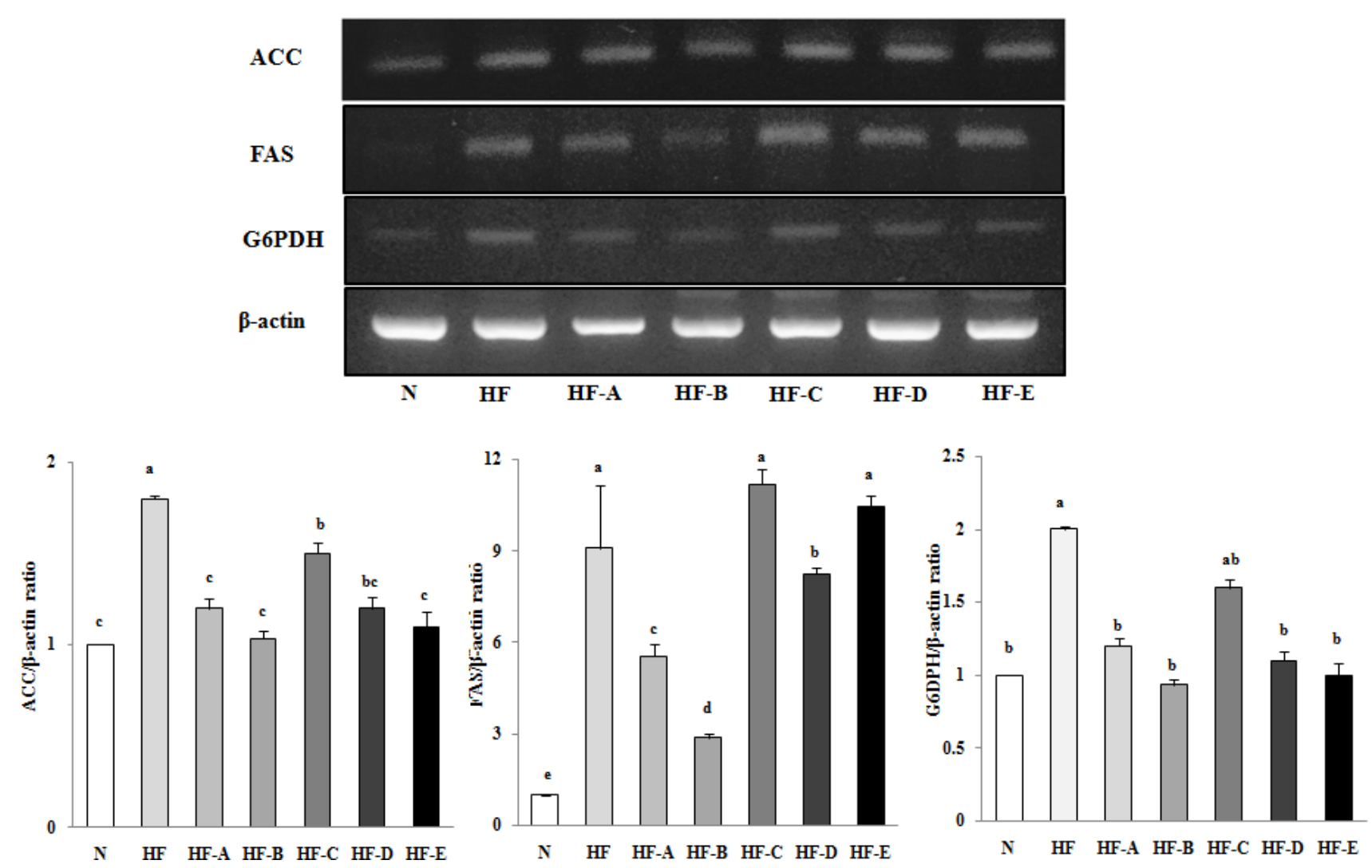

Figure 4. Effects of spirit vinegar and natural fermented vinegar products on the mRNA expression of enzymes related to lipid synthesis in the liver of rats fed a high-fat diet. The mRNA expression of ACC, FAS, and G6PDH was measured by RT-PCR. In the determination of mRNA levels, $\beta$-actin served as a loading control. Values are the mean \pm SE $(n=10)$. Bars with different letters are significantly different at $\mathrm{p}<0.05$ by Tukey's test. N: normal diet group, HF: high-fat diet group, HF-A: high-fat diet + spirit vinegar administered group, HF-B: high-fat diet + apple vinegar administered group, HF-C: high-fat diet + brown rice vinegar administered group, HF-D: high-fat diet + lemon vinegar administered group, and HF-E: high-fat diet + balsamic vinegar administered group

(A)

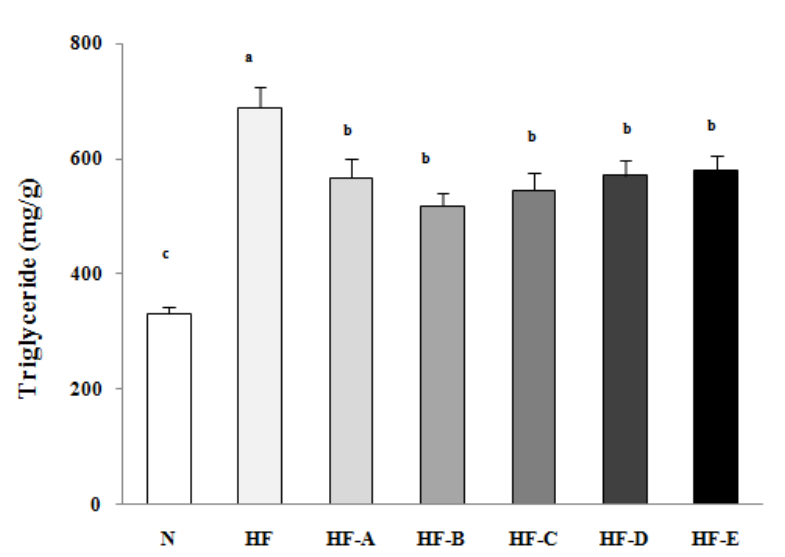

(B)

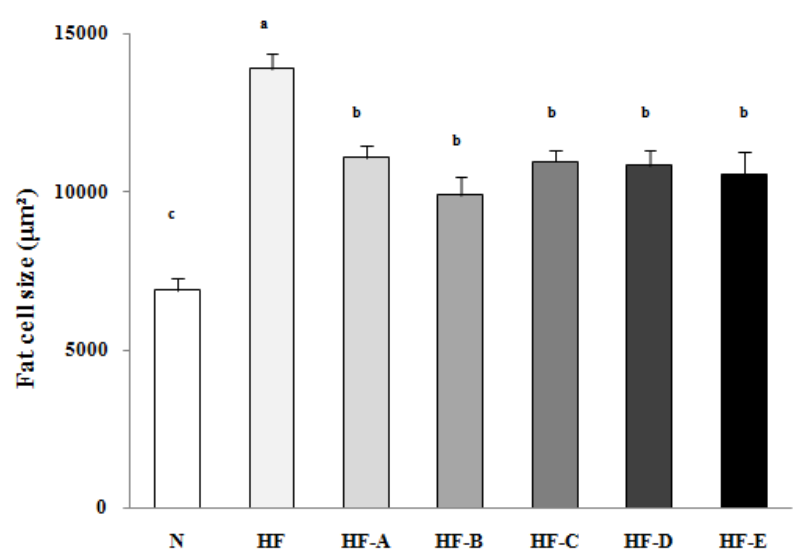

(C)

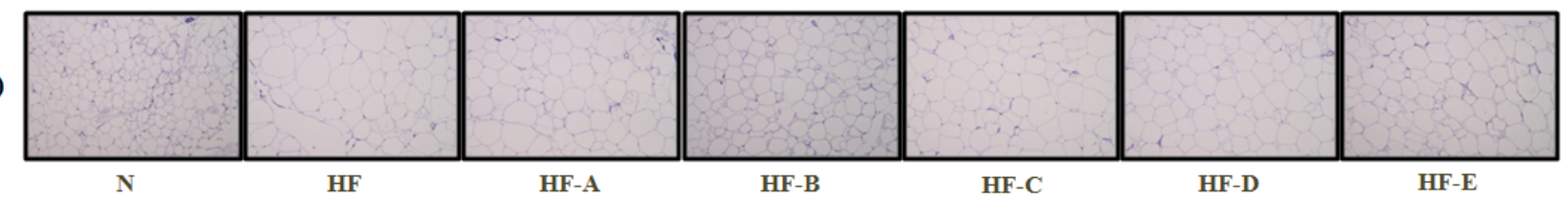

Figure 5. Effects of spirit vinegar and natural fermented vinegar products on epidydimal triglyceride content (A), representative findings (B), and adipocyte size $(\mathrm{C})$ in rats fed a high-fat diet. Epididymal fat tissues were visualized by hematoxylin and eosin staining. Adipocyte size was measured using a microscope and quantified using an image analyzer. Values are the mean $\pm \mathrm{SE}(\mathrm{n}=10)$. Bars with different letters are significantly different at $\mathrm{p}$ $<0.05$ by Tukey's test. N: normal diet group, HF: high-fat diet group, HF-A: high-fat diet + spirit vinegar administered group, HF-B: high-fat diet + apple vinegar administered group, HF-C: high-fat diet + brown rice vinegar administered group, HF-D: high-fat diet + lemon vinegar administered group, and HF-E: high-fat diet + balsamic vinegar administered group 


\section{8. mRNA Expression of Enzymes Related to Lipid Synthesis in the Liver}

To investigate the effect of vinegar on lipid synthesis in liver tissue, we measured the gene expression of ACC, FAS, and G6PDH mRNA, the major enzymes involved in lipid synthesis in the liver (Figure 4). The gene expression of ACC, FAS, and G6PDH mRNA was higher in the HF group than in the $\mathrm{N}$ group. ACC gene expression in the liver was significantly lower in the HF-vinegar administered groups than in the HF group. The expression of the FAS gene was reduced only in the HF-A, HF-B, HF-D, and HF-E groups compared with that in the HF group. G6PDH gene expression in the liver was significantly lower in the HF-vinegar administered groups, except for the HF-D and HF-E groups, than in the HF group.

\subsection{Epididymal Adipose Tissue TG Content and Histological Changes}

The TG contents in epididymal adipose tissues were significantly increased in the HF group compared to those in the $\mathrm{N}$ group (Figure $5 \mathrm{~A}$ ). The TG contents in the HF-vinegar administered groups (HF-A, HF-B, HF-C, HF-D, and HF-E) were significantly lower compared to that in the HF group. The TC content showed no significant difference among the HF groups. Compared to the $\mathrm{N}$ group, the HF group showed hypertrophy of epididymal adipocytes on microscopic evaluation of the size of epididymal adipocytes stained with $\mathrm{H} \& \mathrm{E}$ (Figure $5 \mathrm{~B}$ ) and adipose tissue size analysis using an image analyzer (Figure 5C). The sizes of adipocytes in the HF-vinegar administered groups were significantly decreased compared to those in the HF group, with no significant difference among the HF-vinegar administered groups.

\section{Discussion}

Vinegars prepared with fruits and cereals mainly have a higher content of polyphenolic compounds [10,11,13,28,29], which have been reported to prevent or treat obesity, inhibition of pancreatic lipase activity [39], hyperlipidemia, lipid peroxidation, inflammation, cancer, and hypertension [5,15,40,41,42]. Apple and apple products (vinegar, cider or juice) contain polyphenolic compounds such as chlorogenic acid, benzoic acid, caffeic acid, gallic acid, caftaric acid, syringic acid, coumaric acid, ferulic acid, catechin, epicatechin, tyrosol and vanillin [11]. Lemonbased products are rich in limonoids, coumarins, and flavonoid compounds such as naringin, neohesperidin, and hesperidin [13]. The black vinegar prepared with unpolished rice contains dihydroferulic acid, dihydrosinapic acid, sinapic acid, vanillic acid, and p-hydroxycinnamic acid $[10,28]$. Balsamic vinegar also contains high levels of total polyphenols and flavonoids [29]. According to Etherton et al. [43], biological active compounds improve more and better effective health benefits compared to other nutrients. Several reports have demonstrated that polyphenols and flavonoids compounds exert anti-obesity and hypolipidemic effects [31,39,42,44,45]. Among all polyphenol compounds, chlorogenic acid- or quercetin-containing apple products have been shown to have anti-obesity effects in HFD-induced obesity [30,31]. Fukuchi et al. [46] indicated that citrus bioactive phenolics were reported to promote less body weight gain and white adipose tissue gain in mice after feeding of a HFD. Acetic acid is a major ingredient of vinegar, representing $6 \%$ of vinegar by volume, and it has also been shown to affect metabolic regulation and to have weight-reducing effects and alleviate hyperlipidemia $[17,19,20,26,27]$. Therefore, in this study, we determined whether acetic acid or bioactive compounds, components of vinegar, were had stronger anti-obesity effects. We compared the effects of spirit vinegar and natural fermented vinegars produced from apple, brown rice, lemon, and grape must on the inhibition of adipogenesis in 3T3-L1 cells and on obesity and the lipid profiles of mice fed a HFD.

Accumulation of lipid globules in the cytoplasm is an indication of the differentiation of preadipocytes to adipocytes [47]. In order to investigate the effects of spirit vinegar and natural fermented vinegar products on the formation of lipid globules in 3T3-L1 preadipocytes, the differentiation of preadipocytes was induced, and lipid globules were selectively stained with Oil Red $\mathrm{O}$ and observed under a microscope. As a result, the formation and accumulation of lipid globules were concentration-dependently inhibited in the vinegar treatments given in the non-cytotoxic range, indicating that all vinegars had an inhibitory effect on lipid differentiation at the cellular level. Thus, we selected both spirit vinegar and natural fermented vinegars and implemented further animal studies in rats fed an obesogenic HF diet.

To investigate the anti-obesity effect of vinegars in experimental animals, the effects of vinegar on body weight gain, feed intake, adipose tissue weights, and blood biomarkers were examined in rats fed a HFD. Reducing body and body fat pad weights are important for preventing obesity [48]. The body weight gain was significantly higher in the experimental animals in the HF group than in those in the $\mathrm{N}$ group, suggesting that obesity was induced by the HFD. The feed intake was not different among the HF groups, but the body weight gain was lower in the HF-apple and balsamic vinegar administered groups than in the HF group. Even though the feed intake in the sprit vinegar group was similar to those in the apple and balsamic vinegar groups, body weight gain was prevented by apple or balsamic vinegar administration, and this may have been due to the higher content of total polyphenols in the apple and balsamic vinegars [11,29]. Previous studies have shown that orally administering vinegar rich in polyphenolic compounds lowered body weight gain in experimental animals [21], and human subjects $[19,49]$. In addition, many studies have found that acetic acid also significantly reduced body weight gain in experimental animals [17,19]. However, in this study, body weight gain in the HF-spirit vinegar administered group was lower compared with that in the HF group, and there was no significant difference between the two groups.

Obesity has been reported to negatively affect health not because of body weight gain but because of the increased mass of visceral fat pads located in the abdominal cavity [50]. Total white fat pad weights were lower in the HF-natural fermented vinegar administered groups than in the HF group. In particular, the weight of 
epididymal fat pads was reduced by natural fermented vinegar administration due to the decrease in the size of adipocytes on histological examination as a result of the lowered TG content. Among the vinegar groups, the HF-apple vinegar administered group resulted in the lowest TG level, size of adipocytes, and weight of epididymal fat pads. In addition, the adipocyte size and TG content values were significantly lower in the epididymal adipose tissues in the HF-spirit vinegar administered group than in the HF groups, despite the fact that the HF-spirit vinegar administered group did not exhibit a change in the white fat pads mass. The limitations of current study were that it did not purpose to assay the constituents of the bioactive compounds detected in vinegar that have anti-obesogenic properties. Some researcher indicated that apple vinegar contains chlorogenic acid and quercetin [30,31]; therefore, we hypothesize that the suppressive effects of apple vinegar on HFD-induced lipid accumulation are owing to these polyphenolic compounds. However, the contents of chlorogenic acid and quercetin in the apple vinegar were not measured to confirm these findings.

Leptin is a cytokine that is secreted by adipocytes. It regulates appetite and energy metabolism by suppressing appetite and providing a feeling of satiety, and it is known that obesity increases leptin secretion [27,51]. In general, serum leptin concentration is proportional to body fat mass, and when excess energy accumulates, production is increased; thus, it is widely used as an indicator of obesity [52]. In current study, the body weight gain and white fat pads weight of rats were reduced by vinegar administration, and the serum leptin level was also decreased.

Obesity and the accumulation of visceral fat pads have been reported to alter serum lipid profiles [53]. In this study, the serum TG, TC, and LDL-C levels were increased by the HFD; moreover, in the HF-natural fermented vinegar administered groups, serum TG, TC, and LDL-C levels were lower than those in the HF group. These effects were possibly mediated by the various phytochemicals and organic acids contained in vinegar [15,30,54,55]. Apple cider vinegars manufactured with different technique has a higher content of total polyphenols and chlorogenic acid reduces the oxidation of LDL-C and prevents cardiovascular disease [54]. Several studies have reported that chlorogenic acid in apple cider vinegar had an effect on improving serum lipid metabolism [30,55]. Black vinegar rich in catechin and chlorogenic acid was also shown to suppress serum TG, TC, and LDL-C levels [15]. Acetic acid is also known to be involved in the regulation of lipid metabolism in vivo $[17,19,26]$. Studies on rats [17] and humans [19] have showed that acetic acid decreases serum TG and TC contents. Acetic acid and various types of organic acids contained in vinegar are known to promote appetite, stimulate the use of fatty acids, and inhibit the serum levels of TG and TC by inhibiting 3-hydroxy-3-methylglutaryl-CoA reductase activity, and suppress glycolysis [26]. However, in the present study, the rats administered spirit vinegar (HF-A group) had similar levels of lipids in the serum and liver as the control rats (HF group). Similar to this result, Tong et al. [27] reported that the oral administration of acetic acid did not affect the serum lipid profiles in rats. Several bioactive components of natural fermented vinegar seem to decrease serum lipid contents, which are increased by a HFD. However, the exact mechanism of the action of natural fermented vinegar is not known and needs further investigation.

The liver is the main site for the biosynthesis and metabolism of TG and TC [56]. Fatty liver is caused by the accumulation of fat when lipids, such as synthesized TG, in the liver are not properly metabolized [57]. Obesity and metabolic syndrome are caused by the accumulation of TG in the liver from increased TG synthesis, which is caused by the increased inflow of free fatty acids from a HFD to the liver through the blood [58]. Our results show that compared to the $\mathrm{N}$ group, the HF group developed noticeably higher levels of TG and lipid accumulation in liver tissue and that body weight gain in the HF group was also dramatically increased compared to that in the $\mathrm{N}$ group. Although the liver weight did not differ among groups, we found that treatment with vinegars significantly lowered TG and TC contents, and fat deposits in liver tissues compared to the levels in the HF group. The ALT, AST, ALP, and LDH activities of serum cardiac biomarkers of inflammation and cellular damage decreased in the vinegar administered rats compared to the high fat-fed rats and were not significantly altered among all HF-vinegar administered groups, which showed the probable safety of the tested vinegars. Taken together, these results on fat content and liver enzyme activity indicate an ameliorate effect of vinegar administration on the development of hepatic metabolic dysfuction due to high fat diet.

Enzymes such as ACC, FAS, and G6PDH play major roles in TG synthesis in the liver [17]. In this study, the gene expression of ACC FAS, and G6PDH increased in the $\mathrm{HF}$ group compared to the $\mathrm{N}$ group; however, the administration of vinegar decreased gene expression. This result indicates that lipogenic gene expression is related to the decreases in hepatic TG, lipid accumulation, and liver weight. Yamashita et al. [17] indicated that vinegar causes a reduction in body fat mass because acetic acid in vinegar inhibits the gene expression of lipogenic-related enzymes such as ACC, FAS, and malic enzyme. Thus, acetic acid in vinegar also seems to be involved in gene expression regarding fat synthesis in liver tissue. Our data show that spirit vinegar or natural fermented vinegars lowered high liver TG contents by down-regulating the genes for ACC FAS, and G6PDH and that this was associated with a decrease in the serum TG content.

Our results are consistent with previous researches using polyphenol-rich vinegars and animal treatment tests with persimmon vinegar [14], black vinegar [15,27], tomato vinegar [21], pomegranate vinegar [39], apple cider vinegar [54], pineapple vinegar [59], and fruit vinegar [60], which also shown similar effects as found in current study, including suppression of lipid accumulation and hyperlipidemic effects.

\section{Conclusion}

In conclusion, vinegar products can effectively suppress the differentiation of 3T3-L1 cells. In addition, orally administered vinegar in rats fed a HFD had beneficial effects, including decreases in the weight of visceral fat pads, size of epididymal adipocytes, serum levels of TG and TC, liver weight and hepatic steatosis, and expression of lipogenic genes without affecting appetite and 
cytotoxicity. Spirit vinegar and natural fermented vinegars treatments had anti-obesity effects; however, apple vinegar was the most effective. It is assumed that the antiobesity effects of the vinegars were due to the polyphenolic compounds and/or acetic acid in the vinegar samples. Additional studies are required to further identify the anti-obesogenic active substances and clarify the mechanism of apple vinegar on anti-obesity effects.

\section{Acknowledgments}

This research was supported by a grant funded CJ Cheiljadang Corp. (0F16-KM01-RD), Republic of Korea.

\section{Statement of Competing Interests}

The authors have no competing interests

\section{List of abbreviations}

ACC, acetyl-CoA carboxylase;

ALP, alkaline phosphatase;

ALT, alanine aminotransferase;

AST, aspartate aminotransferase;

FAS, fatty acid synthetase;

G6PDH, glucose-6-phosphate dehydrogenase;

HDL-C, high-density lipoprotein cholesterol;

HFD, high fat diet;

LDH, lactate dehydrogenase;

LDL-C, low-density lipoprotein-cholesterol;

TC, total cholesterol;

TG, triglyceride.

\section{References}

[1] Leung WY, Thomas GN, Chan JC, Tomlinson B. "Weight management and current options in pharmacotherapy: orlistat and sibutramine”, Clinical Therapeutics, 25(1), 58-80, 2003.

[2] Wang YW, Jones PJ. "Conjugated linoleic acid and obesity control: efficacy and mechanisms", International Journal of Obesity, 28(8), 941-955, 2004.

[3] George AB. Louis AT. "Medicinal strategies in the treatment of obesity”, Nature, 404(6778), 672-677, 2000.

[4] Guerrero EDA, Mejias RC, Marin RN, Lovillo MP, Barroso CG. "A new FT-IR method combined with multivariate analysis for the classification of vinegars from different raw materials and production processes", Journal of Science Food and Agriculture, 90(4), 721-728, 2010.

[5] Ho CW, Lazim AM, Fazry S, Zaki UKHH, Lim SJ. "Varieties, production, composition and health benefits of vinegars: A review," Food Chemistry, 221(2017), 1621-1630, 2017.

[6] Lim EJ, Cha GH. "Study on manufacturing of vinegar through literatures of the Joseon dynasty", Journal of the Korean Society of Food Culture, 25(6), 680-707, 2010.

[7] Korea Food Standard Code (KFDA). "Korea Food and Drug Administration”, Cheongwon, Korea, pp5-21, 2012.

[8] Andrés-Barrao C, Saad MM, Cabello Ferrete E, Bravo D, Chappuis ML, Ortega Pérez R, Junier P, Perret X, Barja F. "Metaproteomics and ultrastructure characterization of Komagataeibacter spp. involved in high-acid spirit vinegar production”, Food Microbiology, 55(2016), 112-122, 2016.

[9] Sinclair C. "International Dictionary of Food and Cooking", $1^{\text {st }}$ Edn, Fitzroy Dearborn Publishers, Chicago • London, UK, pp3-5, 1998.
[10] Shimoji Y, Tamura Y, Nakamura Y, Nanda K, Nishidai S, Nishikawa Y, Tanaka T. "Isolation and identification of DPPH radical scavenging compounds in Kurosu (Japanese unpolished rice vinegar)", Journal of Agricultural and Food Chemistry, 50(20), 6501-6503, 2002.

[11] Alonso AM, Castro R, Rodriguez MC, Guillen DA, Barroso CG. "Study of the antioxidant power of brandies and vinegars derived from sherry wines and correlation with their content in polyphenols”, Food Research International, 37(7), 715-721, 2004.

[12] Jeong CH, Choi GN, Kim JH, Kawk JH, Kang ST, Choi SG, Heo HJ. "In vitro antioxidant properties and phenolic composition of Korean commercial vinegars”, Food Science and Biotechnology, 18(5), 1258-1262, 2009.

[13] Gonzalez-Molina E, Dominguez-Perles R, Moreno DA, GarciaViguera C. "Natural bioactive compounds of Citrus limon for food and health", Journal of Pharmaceutical and Biomedical Analysis, 51(2), 327-345, 2010.

[14] Moon YJ, Choi DS, Oh SH, Song YS, Cha YS. "Effects of persimmon-vinegar on lipid and carnitine profiles in mice”, Food Science and Biotechnology, 19(2), 343-348, 2010.

[15] Chou CH, Liu CW, Yang DJ, Wu YH, Chen YC. "Amino acid, mineral, and polyphenolic profiles of black vinegar, and its lipid lowering and antioxidant effects in vivo", Food Chemistry, 168(1), 63-66, 2015.

[16] Vogel RA, Correti MC, Plotnick GD. "The postprandial effect of components of the Mediterranean diet on endothelial function”, Journal of the American College of Cardiology, 36(5), 1455-460, 2000.

[17] Yamashita H, Fujisawa K, Ito E, Idei S, Kawaguchi N, Kimoto M, Hiemori M, Tsuji H. "Improvement of obesity and glucose tolerance by acetate in type 2 diabetic Otsuka Long-Evans Tokushima fatty (OLETF) rats”, Bioscience Biotechnology, and Biochemstry, 71(5), 1236-1243, 2007.

[18] Johnston CS, Quagliano S, White S. "Vinegar ingestion at mealtime reduced fasting blood glucose concentrations in healthy adults at risk for type 2 diabetes", Journal of Functional Foods, 5(4), 2007-2011, 2013.

[19] Kondo T, Kishi M, Fushimi T, Kaga T. “Acetic acid upregulates the expression of genes for fatty acid oxidation enzymes in liver to suppress body fat accumulation”, Journal of Agricultural and Food Chemistry, 57(13), 5982-5986, 2009.

[20] Kondo T, Kishi M, Fushimi T, Ugajin S, Kaga T. "Vinegar intake reduces body weight, body fat mass, and serum triglyceride levels in obese Japanese subjects", Bioscience Biotechnology, and Biochemstry, 73(8), 1837-1843, 2009.

[21] Lee JH, Cho HD, Jeong JH, Lee MK, Jeong YK, Shim KW, Seo KI. "New vinegar produced by tomato suppresses adipocyte differentiation and fat accumulation in 3T3-L1 cells and obese rat model”, Food Chemistry, 141(3), 3241-3249, 2013.

[22] Park YH, Choi JH, Whang K, Lee SO, Yang SA, Yu MH. "Inhibitory effects of lyophilized dropwort vinegar powder on adipocyte differentiation and inflammation”, Journal of Life Science, 24(5), 476-484, 2014.

[23] Kondo S, Tayama K, Tsukamoto Y, Ikeda K, Yamori Y. "Antihypertensive effects of acetic acid and vinegar on spontaneously hypertensive rats”, Bioscience Biotechnology, and Biochemstry, 65(12), 2690-2694, 2001.

[24] Kim H, Lee H, Shin KS. "Intestinal immunostimulatory activity of neutral polysaccharide isolated from traditionally fermented Korean brown rice vinegar", Bioscience Biotechnology, and Biochemstry, 80(12), 2383-2390, 2016.

[25] Nakao C, Yamada E, Fukaya M, Tayama K, Tsukamoto Y, Sato Y. "Effect of acetate on glycogen replenishment in liver and skeletal muscles after exhaustive swimming in rats”, Scandinavian Journal of Medicine \& Science in Sports, 11(1), 33-37, 2001.

[26] Fushimi T, Suruga K, Oshima Y, Fukiharu M, Tsukamoto Y, Goda T. "Dietary acetic acid reduces serum cholesterol and triacylglycerols in rats fed a cholesterol-rich diet”, British Journal of Nutrition, 95(5), 916-924, 2006.

[27] Tong LT, Katakura Y, Kawamura S, Baba S, Tanaka Y, Udono M, Kondo Y, Nakamura K, Imaizumi K, Sato M. "Effects of Kurozu concentrated liquid on adipocyte size in rats", Lipids in Health and Disease, 9, 134-146, 2010.

[28] Sakanaka S, Ishihara Y. "Comparison of antioxidant properties of persimmon vinegar and some other commercial vinegars in radical-scavenging assays and on lipid oxidation in tuna homogenates”, Food Chemistry, 107(2), 739-744, 2008. 
[29] Masinoa F, Chinnici F, Bendini A, Montevecchi G, Antonelli A. "A study on relationships among chemical, physical, and qualitative assessment in traditional balsamic vinegar", Food Chemistry, 106(1), 90-95, 2008.

[30] Cho AS, Jeon SM, Kim MJ, Yeo J, Seo KI, Choi MS, Lee MK. "Chlorogenic acid exhibits anti-obesity property and improves lipid metabolism in high-fat diet-induced-obese mice", Food and Chemical Toxicology, 48(3), 937-943, 2010.

[31] Snyder SM, Zhao B, Luo T, Kaiser C, Cavender G, HamiltonReeves J, Sullivan DK, Shay NF. "Consumption of quercetin and quercetin-containing apple and cherry extracts affects blood glucose concentration, hepatic metabolism, and gene expression patterns in obese C57BL/6J high fat-fed mice”, Journal of Nutrition, 146(5), 1001-1007, 2016

[32] Brimson JM, Brimson SJ, Brimson CA, Rakkhitawatthana V, Tencomnao T. "Rhinacanthus nasutus extracts prevent glutamate and amyloid- $\beta$ neurotoxicity in HT-22 mouse hippocampal cells: possible active compounds include lupeol, stigmasterol and $\beta$ sitosterol”, International Journal of Molecular Sciences, 13(4), 5074-5097, 2012.

[33] Reeves PG, Nielson FH, Fahey Jr GC. "AIN-93 purified diets for laboratory rodents: final report of the American Institute of Nutrition adhoc writing committee on the reformulation of the AIN-76A rodent diet”, Journal of Nutrition, 123(11), 1939-1951, 1993.

[34] Friedwald W, Levy R, Fredrickson D. "Estimation of the concentration of low-density lipoprotein cholesterol in plasma, without use of the preparative ultracentrifuge”, Clinical Chemistry, 18(6), 499-502, 1972.

[35] Folch J, Lees M, Sloane-Stanley G. "A simple method for the isolation and purification of total lipids from animal tissues", Journal of Biological Chemistry, 226(1), 497-509, 1957.

[36] Biggs HG, Erikson TM, Moorehead WR. "A manual colorimetric assay of triglyceride in serum”, Clinical Chemistry, 21(3), 437-441, 1975.

[37] Zlatkis A, Zak B. "Study of a new cholesterol reagent”, Analytical Biochemistry, 29(1), 43-48, 1969.

[38] Nakabayashi H, Hashimoto T, Ashida H, Nishiumi S, Kanazawa $\mathrm{K}$. "Inhibitory effects of caffeine and its metabolites on intracellular lipid accumulation in murine 3T3-L1 adipocytes", Biofactors, 34(4), 293-302, 2008.

[39] Karamaĉ M, Amarowicz R. "Inhibition of pancreatic lipase by phenolic acids: examination in vitro", Zeitschrift fuer Naturforschung Section C Journal of Biosciences, 51(11-12), 903905, 1996.

[40] Ok E. Do GM, Lim Y, Park JE, Park YJ, Kwon O. "Pomegranate vinegar attenuates adiposity in obese rats through coordinated control of AMPK signaling in the liver and adipose tissue”, Lipids in Health and Disease, 12, 163-171, 2013.

[41] Nakajima VM, Macedo GA, Macedo JA. "Citrus bioactive phenolics: Role in the obesity treatment”, LWT - Food Science and Technology, 59(2), 1205-1212, 2014.

[42] Wang S, Moustaid-Moussa N, Chen L, Mo H, Shastri A, Su R, Bapat P, Kwun I, Shen CL. "Novel insights of dietary polyphenols and obesity", The Journal of Nutritional Biochemistry, 25(1), 1-18, 2014.

[43] Etherton PMK, Lefevre M, Beecher GR, Gross MD, Keen CL, Etherton TD. "Bioactive compounds in nutrition and healthresearch methologies for establishing biological function: the antioxidant and anti-inflammatory effects of flavonoids on the atherosclerosis”, Annual Review of Nutrition, 24, 511-538, 2004.

[44] Hsu CL, Yen GC. "Effects of flavonoids and phenolic acids on the inhibition of adipogenesis in 3T3-L1 adipocytes", Journal of Agricultural and Food Chemistry, 55(21), 8404-8410, 2007.
[45] Noratto GN, Martino HS, Simbo S, Byrne D, Mertens-Talcott SU. "Consumption of polyphenol-rich peach and plum juice prevents risk factors for obesity-related metabolic disorders and cardiovascular disease in Zucker rats", Journal of Nutritional Biochemistry, 26(6), 633-641, 2015.

[46] Fukuchi Y, Hiramitsu M, Okada M, Hayashi S, Nabeno Y, Osawa T, Naito M. "Lemon polyphenols suppress diet-induced obesity by up-regulation of mRNA levels of the enzymes involved in betaoxidation in mouse white adipose tissue", Journal of Clinical Biochemistry and Nutrition, 43(3), 201-209, 2008.

[47] Ramirez-Zacarias J, Castro-Munozledo F, Kuri-Harcuch W. "Quantitation of adipose conversion and triglycerides by staining intracytoplasmic lipids with oil red O", Histochemistry, 97(6), 493-497, 1992.

[48] Ho JN, Son ME, Lim WC, Lim ST, Cho HY. “Antiobesity effects of germinated brown rice extract through down-regulation of lipogenesis genes in high fat diet-induced obese mice”, Bioscience Biotechnology, and Biochemistry, 76(6), 1068-1074, 2012.

[49] Hamadate K, Nakamura K, Hirai M, Yamamoto T, Yamaguchi H, Iizuka M, Yamamoto E, Iwama Y, Yazawa K. "Effect of a dietary supplement containing Kurozu (a Japanese traditional health drink) concentrate on several obesity-related parameters in obese Japanese adults: a randomized, double-blind, placebo-controlled trial”, Functional Foods in Health \& Disease, 3(8), 310-322, 2013

[50] Bjorntorp P. "The associations between obesity, adipose tissue distribution and disease”, Journal of Internal Medicine, 723, 121134, 1998.

[51] Friedman JM, Halaas JL. "Leptin and the regulation of body weight in mammals", Nature, 395(6704), 763-770, 1998.

[52] Havel PJ. "Role of adipose tissue in body-weight regulation: mechanism regulating leptin production and energy balance", Proceedings of the Nutrition Society, 59(3), 359-371, 2000.

[53] Morton NM, Paterson JM, Masuzaki H, Holmes MC, Staels B, Fievet C, Walker BR, Flier JS, Mullins JJ, Seckl JR. "Novel adipose tissue-mediated resistance to diet-induced visceral obesity in 11-beta-hydroxysteroid dehydrogenase type 1-deficient mice", Diabetes. 53(4), 931-938, 2004.

[54] Laranjinha JA, Almeida LM, and Madeira VM. "Reactivity of dietary phenolic acids with peroxyl radicals: antioxidant activity upon low density lipoprotein peroxidation”, Biochemical Pharmacology, 48(3), 487-494, 1994.

[55] Budak NH, Kumbul Doguc D, Savas CM, Seydim AC, Kok Tas T, Ciris MI, Guzel-Seydim ZB. "Effects of apple cider vinegars produced with different techniques on blood lipids in highcholesterol-fed rats", Journal of Agricultural and Food Chemistry, 59(12), 6638-6644, 2011.

[56] Browning JD, Horton JD. "Molecular mediators of hepatic steatosis and liver injury", The Journal of Clinical Investigation, 114(2), 147-152, 2004.

[57] Schaefer EJ. "Lipoproteins, nutrition, aging, and atherosclerosis", The American Journal of Clincal Nutrition, 61(3), 726S-740S, 1995.

[58] Buettner R, Parhofer KG, Woenckhaus M, Wrede CE, KunzSchughart LA, Scholmerich J, Bollheimer LC. "Defining high-fatdiet rat models: metabolic and molecular effects of different fat types”, Journal of Molecular Endocrinology, 36(3), 485-501, 2006

[59] Mohamad NE, Yeap SK, Lim KL, Yusof HM, Beh BK, Tan SW, Ho WY, Sharifuddin SA, Jamaluddin A, Long K, Nik Abd Rahman NM, Alitheen NB. "Antioxidant effects of pineapple vinegar in reversing of paracetamol-induced liver damage in mice”, Chinese Medicine, 10, 3-13, 2015.

[60] Bounihi A, Bitam A, BouazzaA, Yargui L, Koceir EA. "Fruit vinegars attenuate cardiac injury via anti-inflammatory and antiadiposity actions in high-fat diet-induced obese rats", Pharmaceutical Biology, 55(1), 43-52, 2017. 